\section{Emergence of Hyper-Resistant} Escherichia coli MG1655 Derivative Strains after Applying Sub-Inhibitory Doses of Individual Constituents of Essential Oils

\author{
Beatriz Chueca ${ }^{1}$, Daniel Berdejo ${ }^{1}$, Nelson J. Gomes-Neto ${ }^{2}$, Rafael Pagán ${ }^{1}$ and \\ Diego García-Gonzalo ${ }^{1 *}$
}

\section{OPEN ACCESS}

Edited by:

Sahdeo Prasad,

The University of Texas MD Anderson

Cancer Center, USA

Reviewed by:

Tineke H. Jones,

Agriculture and Agri-Food Canada,

Canada

Giulia Tabanelli,

CIRI Agroalimntare, Italy Aparna Ramachandran,

Rutgers University, USA

*Correspondence: Diego García-Gonzalo diego.garcia@unizar.es

Specialty section: This article was submitted to Food Microbiology, a section of the journal

Frontiers in Microbiology

Received: 18 December 2015 Accepted: 19 February 2016 Published: 04 March 2016

Citation:

Chueca B, Berdejo D,

Gomes-Neto NJ, Pagán R and García-Gonzalo D (2016) Emergence of Hyper-Resistant Escherichia coli MG1655 Derivative Strains after Applying Sub-Inhibitory Doses of Individual Constituents of Essential

Oils. Front. Microbiol. 7:273. doi: 10.3389/fmicb.2016.00273
${ }^{1}$ Tecnología de los Alimentos, Departamento de Producción Animal y Ciencia de los Alimentos, Facultad de Veterinaria,
Instituto Agroalimentario de Aragón, Universidad de Zaragoza-CITA, Zaragoza, Spain, ${ }^{2}$ Laboratory of Food Microbiology,
Department of Nutrition, Health Sciences Center, Federal University of Paraíba, João Pessoa, Brazil

The improvement of food preservation by using essential oils (EOs) and their individual constituents (ICs) is attracting enormous interest worldwide. Until now, researchers considered that treatments with such antimicrobial compounds did not induce bacterial resistance via a phenotypic (i.e., transient) response. Nevertheless, the emergence of genotypic (i.e., stable) resistance after treatment with these compounds had not been previously tested. Our results confirm that growth of Escherichia coli MG1655 in presence of sub-inhibitory concentrations of the ICs carvacrol, citral, and (+)-limonene oxide do not increase resistance to further treatments with either the same IC (direct resistance) or with other preservation treatments (cross-resistance) such as heat or pulsed electric fields (PEF). Bacterial mutation frequency was likewise lower when those IC's were applied; however, after 10 days of re-culturing cells in presence of sub-inhibitory concentrations of the ICs, we were able to isolate several derivative strains (i.e., mutants) displaying an increased minimum inhibitory concentration to those ICs. Furthermore, when compared to the wild type (WT) strain, they also displayed direct resistance and cross-resistance. Derivative strains selected with carvacrol and citral also displayed morphological changes involving filamentation along with cell counts at late-stationary growth phase that were lower than the WT strain. In addition, co-cultures of each derivative strain with the WT strain resulted in a predominance of the original strain in absence of ICs, indicating that mutants would not out-compete WT cells under optimal growth conditions. Nevertheless, growth in the presence of ICs facilitated the selection of these resistant mutants. Thus, as a result, subsequent food preservation treatments of these bacterial cultures might be less effective than expected for WT cultures. In conclusion, this study recommends that treatment with ICs at sub-inhibitory concentrations should be generally avoided, since it could favor the emergence of hyper-resistant strains. To ascertain the true value of EOs and their ICs in the field of food preservation, further research thus needs to be conducted on the induction of increased transient and stable bacterial resistance via such antimicrobial compounds, as revealed in this study.

Keywords: carvacrol, citral, limonene oxide, mutagenesis, genotypic resistance, filamentation 


\section{INTRODUCTION}

Food-related bacteria have developed strategies to overcome a wide variety of unfavorable environmental conditions encountered during food processing or storage: extremely low or high temperatures (Vidovic et al., 2011, 2012), acid pH and a high amount of salts (McMahon et al., 2007), pulsed electric fields (PEF; Sagarzazu et al., 2013), high hydrostatic pressure (HHP; Hauben et al., 1997), and chemical preservatives (Patrignani et al., 2008; Dubois-Brissonnet et al., 2011). These strategies can lead to an increase in bacterial resistance to the homologous stress agent (direct resistance) as well as to heterologous environmental stresses (cross-resistance; Hengge-Aronis, 2011). An increase in bacterial resistance can follow two different patterns: phenotypic resistance, which is transient and under metabolic control, and genotypic resistance, which results from genetic mutations and thus has a permanent effect (Charpentier and Tuomanen, 2000; Corona and Martínez, 2013).

The development of resistance to antibiotics is usually associated with an inheritable resistance that requires a genetic modification, or its acquisition by horizontal gene transfer (Corona and Martínez, 2013). Kohanski et al. (2010) demonstrated a correlation between the mutagenesis induced by sublethal concentrations of some bactericidal antibiotics and an increase in reactive oxygen species (ROS). This pathway is dependent on $\operatorname{rec} A$, which induces SOS response-mutagenesis (Thi et al., 2011).

Recent studies have suggested that essential oils (EOs) or their individual constituents (ICs) have little impact on the development of antimicrobial resistance (McMahon et al., 2008; Hammer et al., 2012; Luz et al., 2012a,b; Gomes Neto et al., 2015). Although plants and their EOs have been long recognized as a valuable source of medicinal agents (Bakkali et al., 2008), only recently have they been proposed as alternatives to help control spoiling and pathogenic microorganisms in the food industry (Burt, 2004; Friedman, 2015). Carvacrol, citral, and (+)limonene are monoterpenes generally recognized as safe (GRAS) food additives whose antimicrobial activities and mechanisms of inactivation have been examined (Somolinos et al., 2010; AitOuazzou et al., 2013; Chueca et al., 2014a,b; Espina et al., 2014). As described in the case of bactericidal antibiotics by Kohanski et al. (2007), an ROS-dependent mechanism of Escherichia coli inactivation was demonstrated for lethal treatments of carvacrol, citral and (+)-limonene (Chueca et al., 2014a,b). Moreover, RecA-mediated SOS response is a commonly activated pathway in bactericidal antibiotics, carvacrol, citral and (+)-limonene: thus, after sublethal treatments with those ICs, one could expect that a mutagenesis would lead to emergence of hyper-resistant mutants.

Although the prospect that food-related bacteria would develop phenotypic resistance after exposure to sub-inhibitory concentrations of EOs and ICs has been ruled out (Luz et al., 2012a,b; Gomes Neto et al., 2015), studies about the possible induction of genotypic bacterial resistance are still scarce.

IC-induced phenotypic and genotypic resistance to food preservation treatments, both chemical (to ICs or food preservatives) and physical (to heat, PEF or HHP), should be evaluated to ascertain the value of ICs as new antimicrobial agents in this field. The resulting findings could likewise help us design combined food preservation processes more efficiently.

This study's objectives are (a) to investigate the effect of Escherichia coli MG1655 growth in presence of sub-inhibitory concentrations of carvacrol, citral, and (+)-limonene oxide on the development of phenotypic and genotypic resistance to chemical (ICs) and physical treatments (heat and PEF); and (b) to determine the cell morphology and growth properties of resulting hyper-resistant derivative strains.

\section{MATERIALS AND METHODS}

\section{Micro-Organisms and Growth Conditions}

The strain used in this study was Escherichia coli MG1655. The culture was maintained in a cryovial at $-80^{\circ} \mathrm{C}$, from which plates of tryptic soy agar (Oxoid, Basingstoke, Hampshire, England) with $0.6 \%$ yeast extract (Oxoid; TSAYE) were prepared on a weekly basis.

We prepared broth subcultures by inoculating a test tube containing $5 \mathrm{~mL}$ of sterile tryptic soy broth (Oxoid) and $0.6 \%$ yeast extract (TSBYE) with one single colony from a plate. After inoculation, the tubes were incubated overnight at $37^{\circ} \mathrm{C} .250$ mL-flasks containing $50 \mathrm{~mL}$ of TSBYE were inoculated with the resulting subcultures to a final concentration of $10^{5}$ colonyforming units $(\mathrm{CFU}) / \mathrm{mL}$. These flasks were incubated under agitation (130 rpm; Selecta, mod. Rotabit, Barcelona, Spain) at $37^{\circ} \mathrm{C}$ until late-stationary growth phase was reached $(24 \mathrm{~h})$.

\section{Minimum Inhibitory Concentration (MIC)}

MIC against E. coli MG1655 was determined for carvacrol (95\%; Sigma-Aldrich, Steinheim, Germany), citral (95\%; SigmaAldrich), and (+)-limonene oxide (97\%; Sigma-Aldrich) by the tube dilution method with an initial concentration of $10^{5} \mathrm{CFU} / \mathrm{mL}$ (Rota et al., 2004). The highest and lowest concentrations tested were 1750 and $50 \mu \mathrm{l} / \mathrm{L}$ of ICs, respectively. These ICs are practically immiscible in water: therefore, we applied a vigorous shaking method to prepare suspensions (Friedman et al., 2002). For ICs, we also prepared negative controls containing TSBYE plus $1750 \mu \mathrm{l} / \mathrm{L}$ of ICs, and positive controls containing TSBYE with microorganisms at a final concentration of $10^{5} \mathrm{CFU} / \mathrm{mL}$. After $24 \mathrm{~h}$ incubation at $37^{\circ} \mathrm{C}$, the MIC was determined as the lowest concentration of each IC in the presence of which bacteria failed to grow, i.e., at which no visible changes could be detected in the broth medium (Clinical and Laboratory Standards Institute, 2012).

\section{Pre-Exposure of Wild Type (WT) E. coli Strain to ICs to Evaluate the Emergence of Phenotypic Resistance}

The assays to evaluate phenotypic changes in bacterial resistance after exposure of E. coli MG1655 to sub-inhibitory (sub-MIC) concentrations of carvacrol, citral, or (+)-limonene oxide in TSBYE were performed following a procedure described by Gomes Neto et al. (2012). Briefly, $2 \mathrm{~mL}$ of broth subculture were inoculated in $18 \mathrm{~mL}$ of TSBYE containing sub-MIC 
concentrations (between $1 / 2$ and $3 / 4 \times$ MIC) of carvacrol, citral, or ( + )-limonene oxide in tubes and vortexed for $30 \mathrm{~s}$ (initial cell counts at ca. $10^{7} \mathrm{CFU} / \mathrm{mL}$ ). The tubes were incubated under agitation $(130 \mathrm{rpm})$ for $24 \mathrm{~h}$ at $37^{\circ} \mathrm{C}$. After the incubation period, lethal treatments with ICs, heat and PEF were performed, as explained below.

\section{Pre-Exposure of Wild Type (WT) E. coli Strain to ICs to Evaluate the Emergence of Genotypic Resistance}

We selected derivative strains of E. coli MG1655 following the procedure described in Kohanski et al. (2010) for bactericidal antibiotics. An overnight culture of E. coli was diluted 1:10,000 into $50 \mathrm{~mL}$ TSBYE in a $250 \mathrm{~mL}$-flask and grown for $3.5 \mathrm{~h}$ at $37^{\circ} \mathrm{C}$ and $130 \mathrm{rpm}$. This second culture was diluted $1: 3$ into fresh TSBYE containing sub-MIC concentrations (between $1 / 2$ and $3 / 4 \times$ MIC) of carvacrol, citral, or (+)-limonene oxide. Five milliliter of these diluted cultures were grown in tubes for $24 \mathrm{~h}$ at $37^{\circ} \mathrm{C}$ and $130 \mathrm{rpm}$. Each day thereafter for 10 days, cells were diluted 1:1000 into $5 \mathrm{~mL}$ TSBYE in a tube containing a sub-MIC amount of the respective IC and grown for $24 \mathrm{~h}$ at $37^{\circ} \mathrm{C}$ and $130 \mathrm{rpm}$. After 10 days, $0.1 \mathrm{~mL}$-samples of each culture were pour-plated and six colonies were selected for re-growth in fresh TSBYE to verify that the changes in resistance observed were due to stable genotypic alterations in the new strains and to determine their MIC for the selection agent ICs.

The exposure of bacterial cells to an agent that increases mutation beyond the normal rate also raises the chances that cell function might be altered (Giraud et al., 2001). We investigated each IC's mutagenic effect by evaluating the occurrence of mutants resistant to rifampicin as a result of point mutations in the $r p o B$ gene (Gutierrez et al., 2013). To achieve this, we started by diluting an overnight culture of $E$. coli 1:10,000 into $50 \mathrm{~mL}$ TSBYE in a $250 \mathrm{~mL}$-flask; it was grown for $3.5 \mathrm{~h}$ at $37^{\circ} \mathrm{C}$ under agitation $(130 \mathrm{rpm})$. The culture was then diluted 1:3 into fresh TSBYE containing sub-MIC concentrations of carvacrol, citral, or (+)-limonene oxide (at a final concentration of $3 / 4 \times \mathrm{MIC}$ ) or $1 \mathrm{mg} / \mathrm{L}$ ampicillin (Sigma-Aldrich) as a positive control. $20 \mathrm{~mL}$ aliquots (five replicates) of these diluted cultures were grown in $100 \mathrm{~mL}$-flasks for $24 \mathrm{~h}$ at $37^{\circ} \mathrm{C}$ and $130 \mathrm{rpm}$, with absorbance at $595 \mathrm{~nm}$ similar for all cultures, in order to obtain detectable and comparable mutant frequencies (Kohanski et al., 2010). Aliquots of each flask were serially diluted and plated on TSAYE with and without $100 \mathrm{mg} / \mathrm{L}$ rifampicin (Sigma-Aldrich). Plates were incubated for $48 \mathrm{~h}$ at $37^{\circ} \mathrm{C}$, and $\mathrm{CFU}$ were counted. Mutation rates were determined by dividing the number of CFU grown in rifampicin plates (mutation events) by the number of CFU grown in antibiotic-free plates (Rosche and Foster, 2000). Fold change in mutation rate was determined for each IC relative to an untreated control.

\section{Evaluation of Increased Bacterial Resistance \\ Lethal IC Treatments}

Prior to treatment, cultures were centrifuged at $6000 \times g$ for 5 $\mathrm{min}$ and resuspended at a final concentration of $2 \times 10^{7} \mathrm{CFU} / \mathrm{mL}$ in $10 \mathrm{~mL}$ of McIlvaine citrate-phosphate buffer of $\mathrm{pH} 7.0$ with $200 \mu \mathrm{L} / \mathrm{L}$ of carvacrol, $300 \mu \mathrm{L} / \mathrm{L}$ of citral, or $500 \mu \mathrm{L} / \mathrm{L}$ of $(+)$ limonene oxide added. These treatment conditions were chosen according to preliminary results (data not shown). IC treatments were carried out at room temperature. $0.1 \mathrm{~mL}$ samples were taken at determined intervals $(15,20$, or 60 min for carvacrol, citral, or (+)-limonene oxide, respectively) to enumerate survivors.

\section{Lethal Heat Treatments}

Heat treatment was carried out in an incubator (FX Incubator, mod. ZE/FX, Zeulab, Zaragoza, Spain) at $55^{\circ} \mathrm{C}$, with a thermocouple (Ahlborn, mod. Almemo 2450, Holzkirchen, Germany) to monitor heating temperature. Prior to treatment, cultures were centrifuged at $6000 \times \mathrm{g}$ for $5 \mathrm{~min}$ and resuspended at a final concentration of $2 \times 10^{7} \mathrm{CFU} / \mathrm{mL}$ in $450 \mu \mathrm{L}$ of McIlvaine citrate-phosphate buffer of $\mathrm{pH} 7.0$ once temperature had stabilized at $55^{\circ} \mathrm{C}$. These treatment conditions were chosen according to preliminary results (data not shown). After treating bacterial suspensions at that temperature for $26 \mathrm{~min}, 0.1 \mathrm{~mL}$ samples were taken to enumerate the amount of survivors.

\section{Lethal PEF Treatments}

PEF treatments were carried out using equipment that delivers exponential decay pulses, as described by García et al. (2005). High electric field pulses were produced by discharging a set of 10 capacitors (6800 pF; Behlke, C-20C682, Kronberg, Germany) via a thyristor switch (Behlke HTS 160-500SCR) in a batch treatment chamber. The capacitors were charged by a high-voltage DC power supply (FUG, HCK 2500M 35,000, Rosenhein, Germany); a function generator (Tektronix AFG 320, Wilsonville, OR, USA) delivered the on-time signal to the switch. The treatment chamber consisted of a cylindrical plastic tube closed with two polished stainless steel electrodes (Raso et al., 2000). The gap between electrodes was $0.25 \mathrm{~cm}$, and the electrode area was $2.01 \mathrm{~cm}^{2}$. Voltage and electrical intensity were measured with a high voltage probe and a current probe, each connected to an oscilloscope (Tektronix TDS 3012B). Experiments began at room temperature $\left(22 \pm 2^{\circ} \mathrm{C}\right)$, and temperature never exceeded $35^{\circ} \mathrm{C}$ inside the treatment chamber. Prior to this treatment, microorganisms were centrifuged at $6000 \times g$ for $5 \mathrm{~min}$ and resuspended for a final concentration of approximately $2 \times$ $10^{7} \mathrm{CFU} / \mathrm{mL}$ in McIlvaine citrate-phosphate buffer of $\mathrm{pH} 7.0$ while electrical conductivity was adjusted to $2 \mathrm{mS} / \mathrm{cm}$. Next, 0.5 $\mathrm{mL}$ of the samples were introduced in the treatment chamber with a sterile syringe, as described by Raso et al. (2000). Cell suspensions were treated with 50 pulses $(1 \mathrm{~Hz}$, pulse width $2 \mu \mathrm{s}$ ) at $35 \mathrm{kV} / \mathrm{cm}$, corresponding to a specific charge of $4.06 \mathrm{~kJ} / \mathrm{kg}$ per pulse. These treatment conditions were chosen according to preliminary results (data not shown). After treatments, 0.1 $\mathrm{mL}$-samples were taken to enumerate survivors.

\section{Enumeration of Survivors}

After treatment, samples were diluted in phosphate buffered saline, pH 7.3 (Oxoid). Then $0.1 \mathrm{~mL}$-samples were pour-plated onto TSAYE. Plates were incubated at $37^{\circ} \mathrm{C}$ for $24 \mathrm{~h}$. After plate incubation, the colonies were counted with an improved image analysis automatic colony counter (Protos; Analytical Measuring 
Systems, Cambridge, United Kingdom) as described by Condón et al. (1996). Inactivation was expressed as the difference in $\log _{10}$ counts prior and posterior to treatment in each case. The error bars in the figures indicate the mean \pm standard deviations from the data obtained from at least three independent experiments carried out with different microbial cultures.

\section{Determination of Cell Morphology of WT and Derivative Strains}

We observed derivative and WT strain cells grown in absence of ICs with a phase contrast microscope (Nikon Eclipse E400, Nikon Corporation, Tokyo, Japan). Two independent cultures were grown at $37^{\circ} \mathrm{C}$ for $24 \mathrm{~h}$ for each strain. We used a high-resolution AxioCam MRc camera (Carl Zeiss AG, Oberkochen, Germany) to capture digital images that were subsequently processed by ZEN 2010 software (Carl Zeiss AG).

\section{Growth Properties of WT and Derivative Strains}

Growth was monitored with an absorbance microplate reader (Tecan Ltd., Tecan Genios, Seestrasse, Switzerland) to measure bacterial culture absorbance at $595 \mathrm{~nm}$ during incubation. To characterize growth kinetics, absorbance values were fitted using Gompertz model nonlinear regression (Gibson et al., 1988), which in this case can be described as follows:

$$
A(t)=C \times \exp (-\exp (-B \times(t-M)))
$$

where $A(t)$ is the absorbance value in time $t, C$ is the absorbance value in the stationary phase, $B$ is the relative growth rate in point $M$, and $M$ is the time at which the cells reach their maximum growth rate.

\section{Competition Experiments between WT and Derivative Strains}

In a competition experiment to evaluate growth capacity of the mutants as compared with that of the WT (i.e., fitness), each derivative strain was grown along with the WT in the same tube. To differentiate each strain within the co-culture, one of them was marked with a kanamycin resistance cassette. Knockouts of malE were constructed using P1 phage transduction and were derived from an E. coli single-gene knockout library (Baba et al., 2006). Positive P1 transductants were confirmed by acquisition of kanamycin resistance. We generated cells of WT and derivative strains containing the kanamycin cassette so that we could account for fitness effects of the cassette itself. Strain behavior in the competition assays after malE deletion or kanamycin resistance cassette insertion did not present any observable modification (data not shown).

Co-cultures of WT and each derivative strain were produced by diluting overnight cultures $1: 10,000$ into $50 \mathrm{~mL}$ of TSBYE, or TSBYE added with sub-MIC concentrations (between $1 / 2$ and $3 / 4 \times$ MIC) of carvacrol, citral or (+)-limonene oxide, in a 250 $\mathrm{mL}$-flask and grown at $37^{\circ} \mathrm{C}$ and $130 \mathrm{rpm}$. To estimate differences in population fitness via the evaluation of respective population sizes, we plated appropriately diluted samples of the co-culture onto non-selective (TSAYE) and selective growth media (TSAYE with $30 \mathrm{mg} / \mathrm{L}$ of Sigma-Aldrich kanamycin added), thereby allowing the growth of one competitor-the marked strain, resistant to kanamycin-while precluding the growth of the other.

For each competition experiment we computed the derivative strain's relative fitness $\left(W_{d e r}\right.$ ) by comparing the $n$-fold expansion of the derivative and WT strains (Lenski et al., 1991) as follows:

$$
W_{d e r}=\left\{\ln \left[N_{d e r}(T) \times d / N_{d e r}(0)\right] / \ln \left[N_{w}(\mathrm{~T}) \times d / N_{w}(0)\right]\right\}
$$

in which $N(0)$ and $N(\mathrm{~T})$ are the proportions of the WT $\left(N_{w}\right)$ and derivative strain $\left(N_{d e r}\right)$ in the population at the onset $(0)$ and at the end $(\mathrm{T})$ of the competition respectively, and $d$ represents entire population growth during the competition:

$$
d=\{[(\mathrm{CFU} / \mathrm{mL}) \text { of all bacteria at } \mathrm{T}] /[(\mathrm{CFU} / \mathrm{mL}) \text { of all }
$$$$
\text { bacteria at } 0]\}(3
$$

The relative fitness $\left(W_{d e r}\right)$ of a derivative strain is a dimensionless factor that measures the growth rate of the derivative strain compared to the WT within a specific cellular environment.

\section{Statistical Analysis}

Data for the evaluation of the MIC, mutation rate experiments, lethal treatments and competition experiments were obtained from at least three independent experiments carried out with different microbial cultures.

ANOVA and $t$-tests were performed with GraphPad PRISM ${ }^{\circledR}$ (GraphPad Software, Inc., San Diego, USA) and differences were considered significant if $p \leq 0.05$.

\section{RESULTS}

\section{ICs do not Increase Phenotypic Resistance} In order to evaluate the emergence of phenotypic resistance after pre-exposure of WT E. coli MG1655 to ICs, cells were incubated with sub-MIC concentrations of carvacrol, citral, or $(+)$-limonene oxide added to the growth medium until bacterial cultures reached late-stationary growth phase $(24 \mathrm{~h})$. These cells were then treated with the same IC applied during their growth (direct resistance), and no differences were found between the resistance of pre-exposed and non-pre-exposed cells $(p>0.05$; data not shown). Pre-exposed cells were also subjected to lethal treatments with the remaining ICs, as well as with physical food preservation treatments-specifically, heat and PEF (crossresistance). Just as observed in the evaluation of direct resistance, cells did not display increased phenotypic cross-resistance after having been pre-exposed to an IC ( $p>0.05$; data not shown).

\section{Isolation of E. coli Strains with Increased Genotypic Resistance to ICs}

After a 10-day selection with sub-MIC concentrations of carvacrol, citral, and (+)-limonene oxide, we isolated E. coli derivative strains. Six strains from each type of selection were randomly selected and re-cultured in TSBYE. In order to evaluate each derivative strain's increase in resistance, an MIC assay was performed. As shown in Table 1, MICs for WT (i.e., the 
TABLE 1 | Minimum inhibitory concentration (MIC; $\mu$ L/L) of Escherichia coli MG1655 wild type strain (WT) and its derivative strains obtained by selection with carvacrol $\left(\mathrm{CAR}_{1}-\mathrm{CAR}_{6}\right)$, citral $\left(\mathrm{CIT}_{1}-\mathrm{CIT}_{6}\right)$, and (+)-limonene oxide $\left(\operatorname{LIM}_{1}\right.$ to LIM $\left._{6}\right)$.

\begin{tabular}{lccc}
\hline Strains tested & Carvacrol & Citral & (+)-Limonene oxide \\
\hline WT & 200 & 1000 & 750 \\
$\mathrm{CAR}_{1}-\mathrm{CAR}_{3}$ & 600 & n.d. & n.d. \\
$\mathrm{CAR}_{4}-\mathrm{CAR}_{6}$ & 200 & n.d. & n.d. \\
$\mathrm{CIT}_{1}-\mathrm{CIT}_{6}$ & n.d. & $>1750$ & n.d. \\
$\mathrm{LIM}_{1}-\mathrm{LIM}_{6}$ & n.d. & n.d. & 1500 \\
\hline
\end{tabular}

n.d., not determined.

original strain), were $200 \mu \mathrm{L} / \mathrm{L}$ of carvacrol, $1000 \mu \mathrm{L} / \mathrm{L}$ of citral, and $750 \mu \mathrm{L} / \mathrm{L}$ of $(+)$-limonene oxide. On the one hand, after selection with either citral or $(+)$-limonene oxide, the six evaluated colonies showed the same increase in resistance, with MICs of $>1750$ and $1500 \mu \mathrm{L} / \mathrm{L}$ for citral and (+)-limonene oxide, respectively. On the other hand, in the presence of carvacrol as selection agent, only three of the six colonies became more resistant, displaying an MIC of $600 \mu \mathrm{L} / \mathrm{L}$ for that specific IC. From the growth at sub-MIC concentrations of carvacrol, citral, and (+)-limonene oxide, we respectively selected three derivative strains showing increased genotypic resistance: $\mathrm{CAR}_{1} \mathrm{CIT}_{1}$ and $\mathrm{LIM}_{1}$ (which from now on we will call CAR, CIT, and LIM).

In order to evaluate the role of ICs in the emergence of genotypic resistance, we determined mutation rates using a rifampicin-based selection method, focusing on the accumulation of mutations that confer resistance to rifampicin due to a mutagenic agent. We thus compared mutation rates observed in E. coli MG1655 after $24 \mathrm{~h}$ of growth in TSBYE in presence and absence of ICs. Firstly, this strain displayed a spontaneous frequency of rifampicin-resistant mutants of nearly $2 \times 10^{-7}$, meaning that 2 out of $10^{7}$ cells had developed resistance to rifampicin. Mutant frequencies after incubation with carvacrol, citral and (+)-limonene oxide were at least 10 times lower than the frequency observed in absence of any IC (Figure 1). However, presence of ampicillin entailed a 10-fold increase in mutant frequency.

\section{Evaluation of Direct- and Cross-Resistance of Selected Derivative Strains to Lethal Treatments with Carvacrol, Citral, (+)-Limonene Oxide, Heat, and PEF}

Once derivative strains had been selected, we evaluated their resistance to several chemical compounds (ICs) and to other physical food preservation technologies (heat and PEF) in citratephosphate buffer of $\mathrm{pH} 7.0$ with an initial concentration of $2 \times 10^{7} \mathrm{CFU} / \mathrm{mL}$. Figure $2 \mathrm{~A}$ shows the inactivation of WT and derivative strains provoked by a treatment with $200 \mu \mathrm{L} / \mathrm{L}$ of carvacrol. A similar inactivation of ca. $5 \log _{10}$ cycles was observed for WT, CAR and LIM $(p>0.05)$. However, the CIT strain was more resistant than the others $(p \leq 0.05)$.

Regarding resistance against $300 \mu \mathrm{L} / \mathrm{L}$ of citral, the three derivative strains showed a higher resistance than the WT strain $\left(p \leq 0.05\right.$; Figure 2B). While nearly $4 \log _{10}$ cycles of the WT

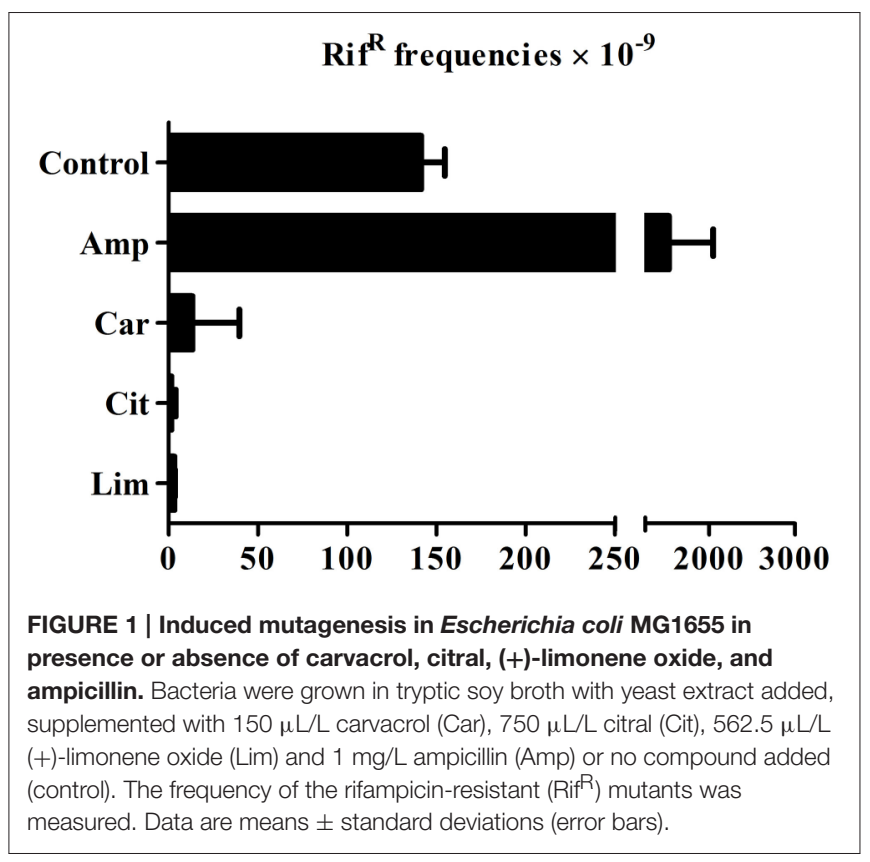

strain had been inactivated after 20 min, only ca. $2 \log _{10}$ cycles of CAR, CIT, and LIM cells were killed under the same conditions.

All of the derivative strains obtained in this study were more resistant to a $60 \mathrm{~min}$-treatment with $500 \mu \mathrm{L} / \mathrm{L}$ of $(+)$-limonene oxide than the WT strain ( $p \leq 0.05$; Figure $2 \mathrm{C}$ ). This treatment inactivated $5 \log _{10}$ cycles of WT cells, but only $2 \log _{10}$ cycles of the initial population of CAR, CIT, and LIM cells.

As can be seen in Figure 2D, the CAR, CIT, and LIM strains were more heat-resistant than the WT strain after a 26min-treatment at $55^{\circ} \mathrm{C}(p \leq 0.05)$. Inactivation due to heat surpassed the detection limit $\left(5 \log _{10}\right.$ cycles) in the WT strain, whereas CAR, CIT, and LIM displayed around $4 \log _{10}$ cycles of inactivation.

Evaluation of PEF resistance showed that ca. $2 \log _{10}$ cycles of initial bacterial populations of WT, CAR, and LIM strains were inactivated after 50 pulses at $35 \mathrm{kV} / \mathrm{cm}(p>0.05)$. Inactivation of the derivative strain CIT by PEF was $1 \log _{10}$ cycle higher than that of the WT strain ( $p \leq 0.05$; Figure 2E).

\section{Determination of Cell Morphology and Growth Properties of the Hyper-Resistant Derivative Strains}

Microscopic comparison of the hyper-resistant derivative strains with the WT strain revealed differing morphologies in the CAR and CIT cell populations, but not in the LIM strain. As seen in Figure 3, around 5\% of CAR and CIT cells were longer, yet similar in width to the WT cells, a phenomenon commonly known as filamentation.

As shown in Table 2, although growth rates for all strains were similar $(p>0.05)$, CFU counts in late-stationary growth phase were lower for single cultures of CAR and CIT strains $\left(\leq 1 \times 10^{9} \mathrm{CFU} / \mathrm{mL}\right)$, amounting to at least half of the cell counts obtained for WT and LIM strains $\left(2 \times 10^{9} \mathrm{CFU} / \mathrm{mL}\right)$. In order to 

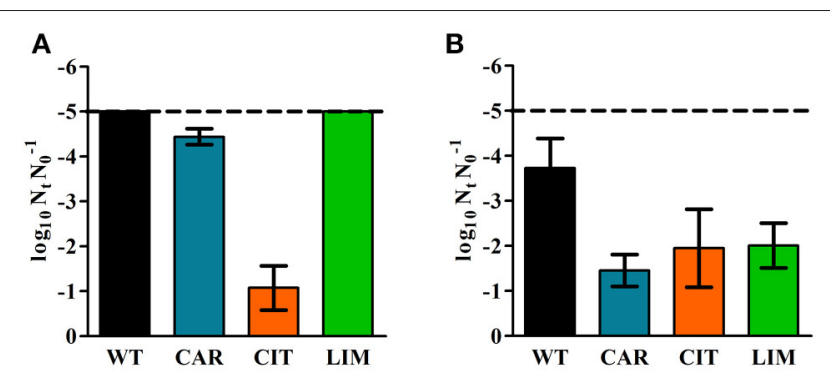

C

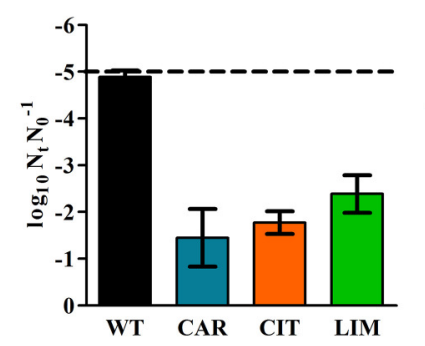

D

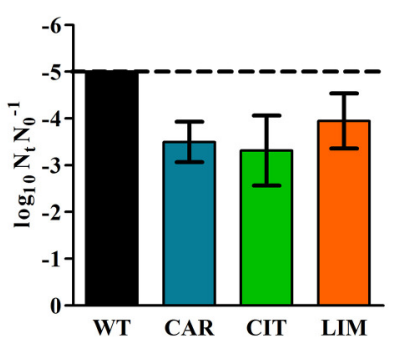

E

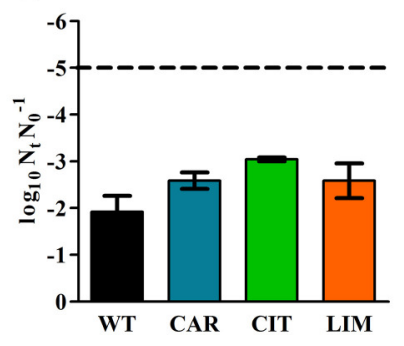

FIGURE 2 | Inactivation of Escherichia coli MG1655 wild type strain (WT) and its derivative strains, obtained by selection with carvacrol (CAR), citral (CIT) and (+)-limonene oxide (LIM), after lethal treatments with: $200 \mu \mathrm{L} / \mathrm{L}$ of carvacrol during $15 \mathrm{~min}(\mathrm{~A}) ; 300 \mu \mathrm{L} / \mathrm{L}$ of citral during $20 \mathrm{~min}(\mathrm{~B}) ; 500 \mu \mathrm{L} / \mathrm{L}$ of (+)-limonene oxide during $60 \mathrm{~min}$ (C); heat treatment at $55^{\circ} \mathrm{C}$ during $26 \mathrm{~min}(\mathrm{D})$; and a PEF treatment at $35 \mathrm{kV} / \mathrm{cm}$ for $\mathbf{5 0}$ pulses (E). Treatment medium was citrate-phosphate buffer of $\mathrm{pH}$ 7.0. Data are means \pm standard deviations (error bars).

learn more about the growth properties of derivative strains, we evaluated co-cultures of WT with each derivative strain starting at $10^{5} \mathrm{CFU} / \mathrm{mL}$ for each strain in liquid growth medium in absence of ICs (Figures 4A,C,E). Statistical differences $(p \leq$ 0.05) in cell counts between WT strain and both CAR and CIT emerged starting with 1 and $7 \mathrm{~h}$ of incubation respectively (Figures 4A,C).

Figures 4B,D,F represent the proportion of WT and derivative strains in each co-culture. Whereas LIM and WT proportions after $10 \mathrm{~h}$ of co-culture were similar (Figure 4F), CAR and CIT cells represented only 0.02 and $0.08 \%$ of the final population respectively (Figures 4B,D). The relative fitness $W_{\text {der }}$ of a mutant is a dimensionless factor that measures the mutant's growth rate compared to WT within a specific cellular environment. Table 3 shows that, whereas $W_{d e r}$ for LIM is nearly 1 (indicating that LIM and WT display similar relative fitnesses), CAR and CIT strains are less efficient than WT when cultured together.
However, when carvacrol at sub-MIC concentrations was added to the growth medium, relative fitness of CAR was not significantly affected $(p>0.05)$. In contrast to results observed for CAR, co-culture of CIT and WT under the presence of citral revealed that the CIT strain's fitness was higher than that of WT cells $(p \leq 0.05)$, with CIT becoming the major strain in that co-culture and displaying a $W_{d e r}$ of 1.36 (Table 3). Although $W_{d e r}$ of the LIM strain was likewise higher in presence of $(+)$ limonene oxide, its increase was lower than $W_{\text {der }}$ of the CIT strain. Consequently, LIM would also out-compete the WT strain in presence of the IC used during the selection of the hyperresistant derivative strain.

\section{DISCUSSION}

This study has demonstrated, for the first time, the emergence of hyper-resistant strains after exposure to the ICs carvacrol, citral, and (+)-limonene oxide. Their resistance was stable after regrowth in absence of the antimicrobial compounds, thus it can be attributed to genotypic modifications of the original strain. In addition, to the best of our knowledge, this is the first report of bacterial resistance against lethal treatments with $(+)$-limonene oxide.

At the onset, increased phenotypic resistance was evaluated after $24 \mathrm{~h}$ bacterial growth in the presence of sublethal concentrations of carvacrol, citral and (+)-limonene oxide. No changes in resistance to either chemical (ICs) or physical (heat and PEF) lethal treatments were observed, in agreement with previous studies (McMahon et al., 2008; Hammer et al., 2012; Luz et al., 2012a,b; Gomes Neto et al., 2015). As a consequence, no increased phenotypic direct- or cross-resistance was detected after pre-exposing E. coli cells to sub-MIC concentrations of ICs for $24 \mathrm{~h}$.

Therefore we proceeded to evaluate the emergence of genotypic or stable resistance after a 10-day selective exposure to sub-MIC concentrations of ICs. Sub-MIC concentrations of bactericidal antibiotics increased intracellular ROS production (Kohanski et al., 2007), leading to activation of SOS response which caused mutagenesis dependent on RecA activity (Kohanski et al., 2010; Thi et al., 2011). Since ROS were involved in the mechanisms of bacterial inactivation via lethal treatments with carvacrol, citral, and (+)-limonene (Chueca et al., 2014a,b), a mutagenic activity similar to the one usually triggered by bactericidal antibiotics was expected. It should be noted that spontaneous mutations occur at random during bacterial growth under optimal conditions (e.g., in absence of ICs). Evaluation of frequency of mutation for our WT strain in absence of ICs (Figure 1) showed similar values than for ME12, an MG1655 derivative (Thi et al., 2011). We also confirmed that ampicillin increased mutation frequency of WT as described by Kohanski et al. (2010). Contrary to what was expected, none of the tested ICs increased bacterial mutagenesis when compared with optimal growth conditions. A lower mutation frequency in presence of ICs would suggest a protective role of those ICs at sub-MIC concentration-contrary to the expected effect of ICs, which, at lethal concentrations, would activate 

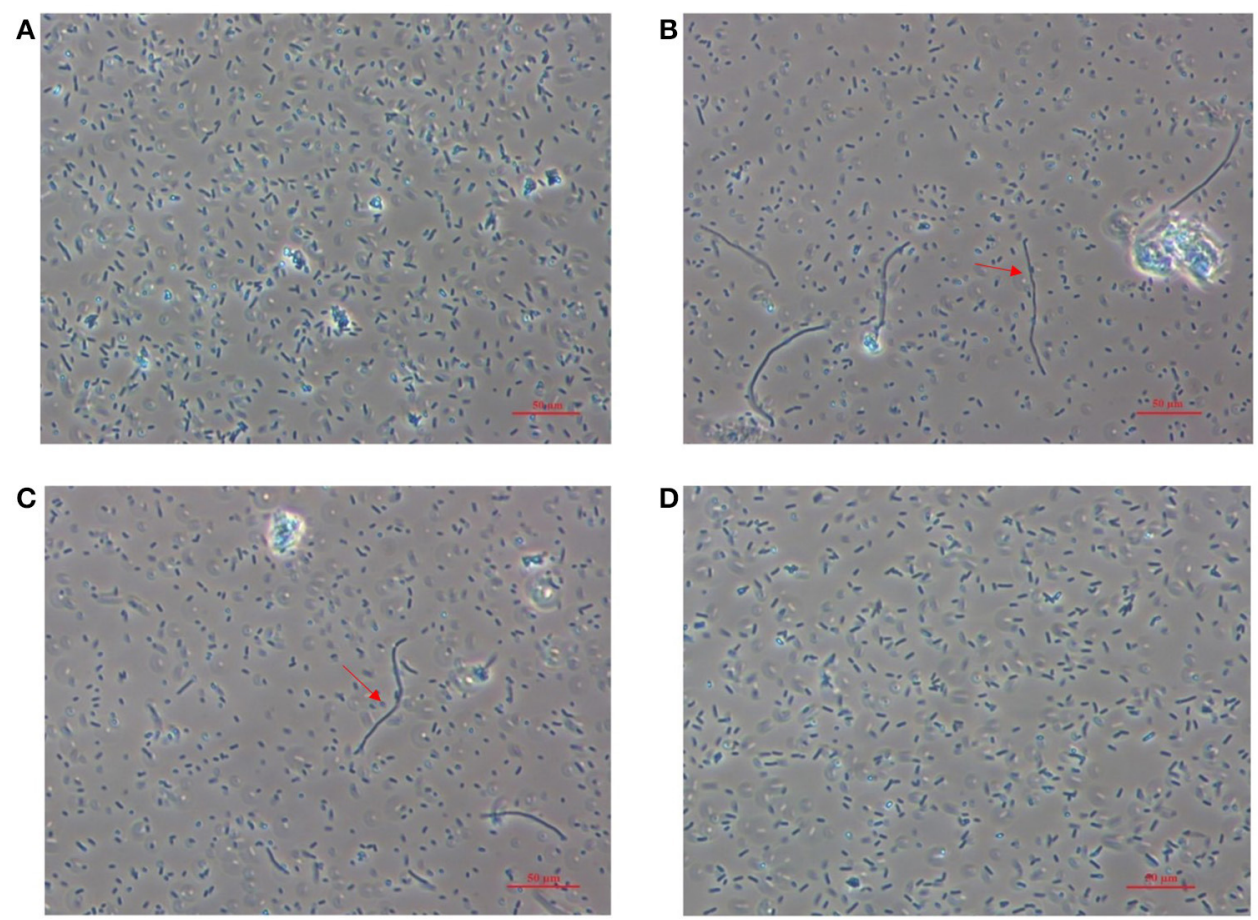

FIGURE 3 | Microscopic image obtained from bacterial cultures of Escherichia coli MG1655 wild type strain (A) and its derivative strains, obtained by selection with carvacrol (B); citral (C); and (+)-limonene oxide (D). Cultures were obtained after $24 \mathrm{~h}$ of incubation at $37^{\circ} \mathrm{C}$ in tryptic soy broth with yeast extract added. Arrows indicate examples of the morphology of filamentous cells.

TABLE 2 | Growth rates and late-stationary phase CFU/mL counts for Escherichia coli MG1655 wild type strain (WT) and its derivative strains obtained by selection with carvacrol (CAR), citral (CIT), and (+)-limonene oxide (LIM) grown at $37^{\circ} \mathrm{C}$ for $24 \mathrm{~h}$ in tryptic soy broth with yeast extract added.

\begin{tabular}{lcc}
\hline Strain & Growth rate & Late-stationary growth phase (24 h) CFU/mL \\
\hline WT & $1.06 \pm 0.14^{1}$ & $2.45 \times 10^{9} \pm 2.95 \times 10^{8 \mathrm{a}}$ \\
CAR & $0.86 \pm 0.12^{1}$ & $1.61 \times 10^{9} \pm 2.14 \times 10^{8 \mathrm{~b}}$ \\
CIT & $1.45 \pm 0.50^{1}$ & $8.41 \times 10^{8} \pm 9.06 \times 10^{7 \mathrm{c}}$ \\
LIM & $1.31 \pm 0.30^{1}$ & $2.26 \times 10^{9} \pm 3.19 \times 10^{8 \mathrm{a}}$
\end{tabular}

Data are means of three independent observations \pm standard deviations. ${ }^{a, b, c}$ same letters or ${ }^{1}$ numbers indicate non-significant differences among mean values; $p>0.05$. Growth rates and CFU/mL were studied independently.

an SOS response (Chueca et al., 2014a,b). Indeed, although the mechanism of bacterial inactivation through carvacrol, citral and (+)-limonene is related to ROS production at lethal concentrations (Chueca et al., 2014a,b) these compounds are also regarded as natural antioxidants (Brewer, 2011). Likewise, LlanaRuiz-Cabello et al. (2015) demonstrated that high concentrations of carvacrol induce oxidative stress in the Caco-2 cell line, but low concentrations of the same IC reverse oxidative damage. Consequently, the observed protection from mutation which is conferred by those ICs could be related to their antioxidant activity at the low sub-MIC concentrations which were applied.
However, after 10 days of continuous exposure to sub-MIC concentrations of each IC, we isolated several E. coli MG1655 derivative strains which displayed an increased MIC to the selective agent (Table 1); we proceeded to characterize three of those strains (CAR, CIT, and LIM) in further detail. According to Fridman et al. (2014), bacterial strategies against chemical agents such as antibiotics could be divided into those which allow a microorganism to grow in the constant presence of an antibiotic at low concentrations (e.g., higher MICs for inhibition assays), and those strategies permitting a microorganism to survive at high antibiotic concentrations but during a limited time (e.g., a higher survival rate from inactivation treatments). Thus, in order to improve our description of the effects of mutations, we not only verified the increase in MICs of derivative strains and evaluated their resistance to lethal stresses of the same IC applied during selection (direct resistance), but we also measured their resistance to the other ICs as well as to physical treatments (cross-resistance).

We started by evaluating increased direct resistance. On the one hand, when compared with the WT strain, the CIT and LIM strains displayed an increased resistance to lethal treatments with citral and (+)-limonene oxide respectively. On the other hand, although the CAR strain had an increased MIC to carvacrol, it was not more resistant to a lethal treatment with carvacrol than the WT strain (Figure 2A). This situation could indicate that the mechanism of action of carvacrol microbial in inhibition assays might differ from its performance in inactivation treatments. 

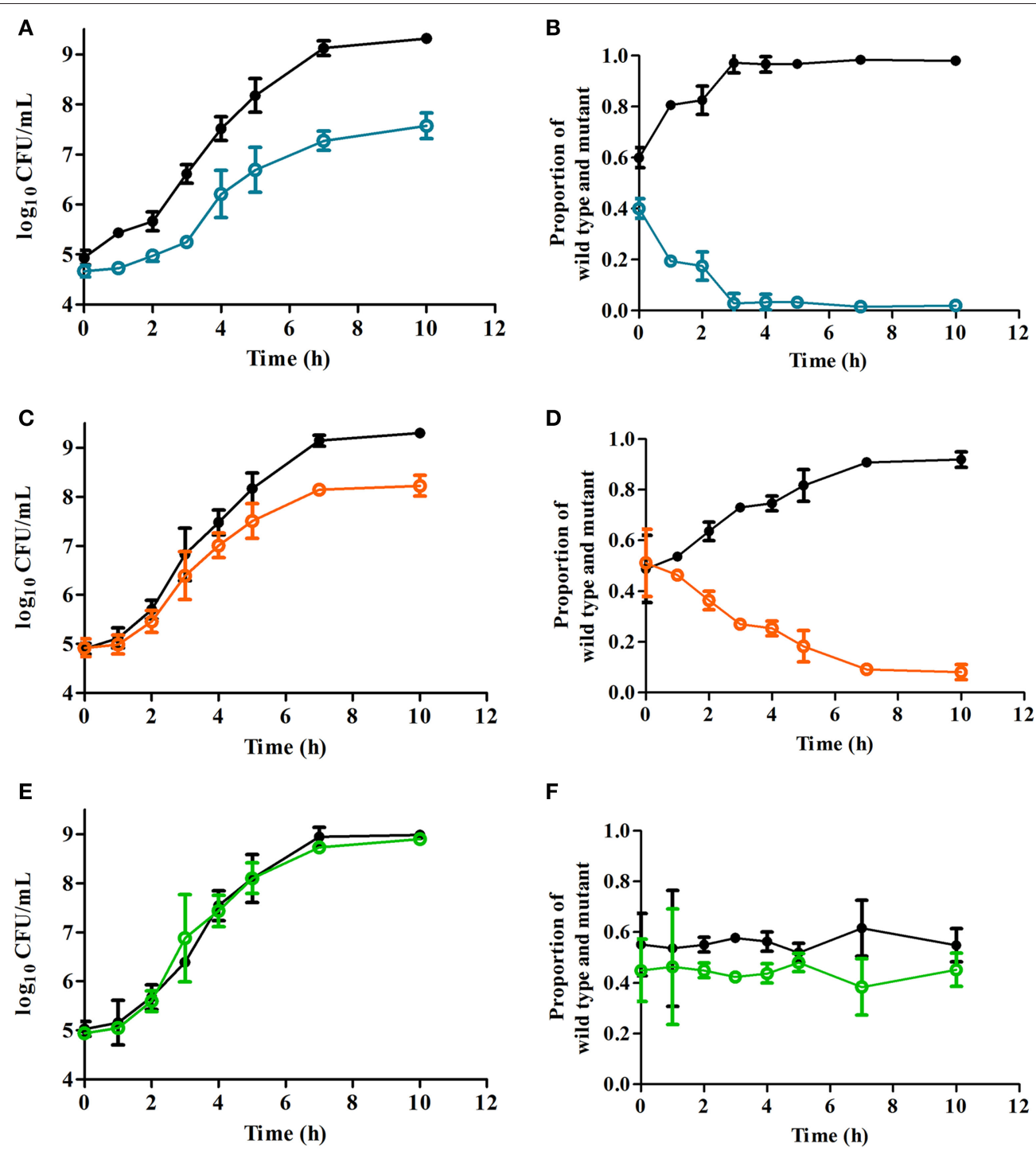

FIGURE 4 | Growth curves measured as $\log _{10} \mathrm{CFU} / \mathrm{mL}(\mathrm{A}, \mathrm{C}, \mathrm{E})$ and proportion of strains within the entire population (B,D,F) of co-cultures with Escherichia coli MG1655 wild type strain (•) and its derivative strains obtained by selection with carvacrol (O, A,B); citral (O, C,D); or (+)-limonene oxide (O, E,F). Cultures were obtained at $37^{\circ} \mathrm{C}$ in tryptic soy broth with yeast extract added. Data are means \pm standard deviations (error bars).

We then proceeded to assess the development of crossresistance in derivative strains. First of all, the three derivatives' heat resistance was likewise increased. All three derivative strains displayed increased resistance against citral and (+)limonene oxide, but only one of them (CIT) developed a higher resistance to carvacrol. However, CAR and LIM derivative strains showed a PEF resistance similar to that observed in the WT strain $(p>0.05)$, and CIT was even less PEF-resistant than the WT $(p \leq 0.05)$. Hauben et al. (1997) correlated the increased heat resistance of barotolerant $E$. coli mutants with similarities between the cellular targets of lethal pressure and heat treatments. Likewise, increase of heat resistance in ICresistant strains could be related to bacterial envelopes, which are regarded as these preservation technologies' primary targets: heat permeabilizes the outer and cytoplasmic membranes (Tsuchido et al., 1985; Mackey et al., 1991); citral can cause sublethal injuries in the outer and inner membranes of E. coli (Somolinos et al., 2010); (+)-limonene can damage the lipopolysaccharide fraction of the cell wall along with the proteins and phospholipids of the outer and inner membranes (Espina et al., 2013); carvacrol, finally, has the capability of interacting with both the lipid bilayer and proteins of the inner and outer membranes (Burt et al., 2007; Hyldgaard et al., 2012; Ait-Ouazzou et al., 2013). Although the mode of action of PEF also targets cell envelopes (García et al., 2005; Chueca et al., 2015), the survival benefits conferred by mutation against ICs and heat-but not against $\mathrm{PEF}$ - could have something to do with the repair and/or higher resistance displayed by cell envelope structures which 
TABLE 3 | Relative fitness ( $W_{\text {der }}$ ) of Escherichia coli MG1655 derivative strains obtained by selection with carvacrol (CAR), citral (CIT) and (+)-limonene oxide (LIM).

\begin{tabular}{lcc}
\hline Co-culture & $\boldsymbol{w}_{\text {der }}$ & $\boldsymbol{w}_{\boldsymbol{d e r}}$ (ICs) \\
\hline $\mathrm{WT}+\mathrm{CAR}$ & $0.66^{\mathrm{a}} \pm 0.04$ & $0.74^{\mathrm{a}} \pm 0.15$ \\
$\mathrm{WT}+\mathrm{CIT}$ & $0.75^{\mathrm{a}} \pm 0.01$ & $1.36^{\mathrm{b}} \pm 0.05$ \\
$\mathrm{WT}+$ LIM & $0.95^{\mathrm{a}} \pm 0.05$ & $1.10^{\mathrm{b}} \pm 0.06$
\end{tabular}

Cells from derivative strains were grown in co-culture with wild type cells (WT) at $37^{\circ} \mathrm{C}$ for $10 \mathrm{~h}$ in tryptic soy broth with yeast extract added, in absence $\left(W_{\text {der }}\right.$ ) and presence of the same individual constituent (ICs) used as selection agents $W_{\text {der }}$ (ICs)]. Data are means of three independent observations \pm standard deviations. Co-cultures involving the same strains were studied independently, thus ${ }^{a, b}$ same letters in each line indicate non-significant differences among mean values; $p>0.05$.

are not targetted by PEF treatments. Along with damage to the bacterial membrane, heat also causes multitarget damages such as ribosome destabilization, enzyme denaturation, and DNA damage, among others (Mackey et al., 1991); carvacrol, moreover, could act as a transmembrane carrier of monovalent cations, fomenting the exchange of hydrogen and potassium ions between the cytoplasm and the external environment (Ultee et al., 2002) and could cause disruption to cellular metabolism and energy production (Chan et al., 2013). Consequently, the potential damage of cytoplasmic structures due to ICs and heat could lead to an absence of increase in PEF resistance (which seems to be exclusively and location-specifically related to membranes: cf. Chueca et al., 2015). In addition, one should note that the advantages conferred by mutations under certain conditions could represent a disadvantage under others-as observed for PEF resistance in CIT cells. Mutations could confer increased resistance to ICs and heat, leading to side-effects in PEF targets such as metabolic costs, higher exposure of the cytoplasmic membrane or a decreased ability to repair that type of damage.

The emergence of antibiotic resistance generally involves the development of specific mutations that enable bacteria to continue to grow in the presence of antibiotics (Cantón and Morosini, 2011); otherwise, it is connected with the selective advantage provided by naturally occurring mutants already present in the population before treatment (Collignon, 2002). Therefore, and, as previously researched in antibiotic-resistant strains (Renzoni et al., 2011), whole genome sequencing of derivative strains would permit the identification of single nucleotide polimorfisms (SNPs) and insertions or deletions (Indels) in those hyper-resistant strains in comparison with WT. Such valuable information would help to increase our knowledge of the mechanisms of bacterial inactivaction via carvacrol, citral, and $(+)$-limonene oxide. Furthermore, identification of these genotypic alterations would help us identify key structures and/or metabolic pathways involved in bacterial resistance to PEF. In addition, it is very likely that the results observed for the nonpathogenic E. coli strain used in this investigation would be similar to those that could occur in the case of pathogenic E. coli strains such as O157:H7.

Phase-contrast microscopial observation of hyper-resistant strains revealed filament formation in a small population (ca.
$5 \%$ of total cells) of CAR and CIT strains (Figure 3). These changes in cell morphology appear to be a general response against stresses (Jones et al., 2013) such as treatments with antibiotics (Thi et al., 2011) and low temperatures (Visvalingam et al., 2012) which inhibit cell division or modify gene and protein expression in filamentous cells (Bereksi et al., 2002; Kieboom et al., 2006). Interestingly, previous publications have associated filamentation with slow growth such as that which we noted in the CAR and CIT strains (Figure 3). As observed for LIM cells, filamentation does not seem to be a pre-requisite for increased resistance. Nevertheless, the resistance displayed by these filamentous strains requires further research.

In addition, co-culture of CAR and CIT strains with the WT strain revealed a predominance of the original strain in absence of ICs (Figures 4A,C), indicating that these mutants would not outcompete the WT strain under optimal growth conditions. Consequently, although the emergence of stable strains that are hyper-resistant to food preservation technologies was confirmed in this study, it seems unlikely that mutant populations of CAR and CIT would take over the entire population under optimal growth conditions. Co-culture of LIM and WT strains nevertheless revealed a similar proportion of both strains in the final population (Figure 4F). Likewise, growth in presence of ICs favored the selection of CIT and LIM strains over the WT strain (Table 3), thus increasing the final proportion of hyperresistant bacteria in the whole population. This could explain the high proportion of mutant cells we observed after 10 days of culturing cells in presence of sub-MIC concentrations of each IC (Table 1). Although ICs lowered the mutation frequency, incubation with sub-MIC concentrations of these antimicrobial compounds would act by selecting in favor of hyper-resistant strains generated during bacterial growth rather than by promoting their generation. As a result, food preservation treatments could be less effective if applied sequentially after treatment with sublethal concentrations of EOs or their ICs.

In conclusion, contrary to previous studies suggesting that EOs or their ICs had little impact on the development of antimicrobial resistance and susceptibility (McMahon et al., 2008; Hammer et al., 2012; Luz et al., 2012a,b; Gomes Neto et al., 2015), our results highlight the importance of preventing the emergence of hyper-resistant strains when using these compounds as antimicrobial agents. Thus, the application of low concentrations (sub-MIC) of EOs and their ICs aiming to slow bacterial multiplication prior to lethal preservation treatments should be avoided. This research demonstrates that the development of genotypic resistance to ICs has important consequences for the practical use of these antimicrobial compounds in food preservation.

\section{AUTHOR CONTRIBUTIONS}

Conceived and designed the experiments: BC, RP, DG. Performed the experiments: BC, DB, NN. Analyzed the data: BC, DB, RP, DG. Wrote the paper: BC, RP, DG. 


\section{ACKNOWLEDGMENTS}

This study received financial support from CICYT (Spanish Interministerial Commission of Science and Technology, Project No. AGL2012-32165 and AGL2015-69565-P), from FEDER, the European Social Fund, from the Aragonese Office of Science, Technology and University Research; and from the Spanish Ministry of Sports, Culture and Education: the latter provided

\section{REFERENCES}

Ait-Ouazzou, A., Espina, L., Gelaw, T. K., De Lamo-Castellví, S., Pagán, R., and García-Gonzalo, D. (2013). New insights in mechanisms of bacterial inactivation by carvacrol. J. Appl. Microbiol. 114, 173-185. doi: 10.1111/jam.12028

Baba, T., Ara, T., Hasegawa, M., Takai, Y., Okumura, Y., Baba, M., et al. (2006). Construction of Escherichia coli K-12 in-frame, single-gene knockout mutants: the Keio collection. Mol. Syst. Biol. 2, 2006.0008. doi: 10.1038/msb41 00050

Bakkali, F., Averbeck, S., Averbeck, D., and Idaomar, M. (2008). Biological effects of essential oils - A review. Food Chem. Toxicol. 46, 446-475. doi: 10.1016/j.fct.2007.09.106

Bereksi, N., Gavini, F., Bénézech, T., and Faille, C. (2002). Growth, morphology and surface properties of Listeria monocytogenes Scott A and LO28 under saline and acid environments. J. Appl. Microbiol. 92, 556-565. doi: 10.1046/j.13652672.2002.01564.x

Brewer, M. S. (2011). Natural antioxidants: sources, compounds, mechanisms of action, and potential applications. Compr. Rev. Food. Sci. Food Saf. 10, 221-247. doi: $10.1111 / j .1541-4337.2011 .00156 . x$

Burt, S. (2004). Essential oils: their antibacterial properties and potential applications in foods-a review. Int. J. Food Microbiol. 94, 223-253. doi: 10.1016/j.ijfoodmicro.2004.03.022

Burt, S. A., Van Der Zee, R., Koets, A. P., De Graaff, A. M., Van Knapen, F., Gaastra, W., et al. (2007). Carvacrol induces heat shock protein 60 and inhibits synthesis of flagellin in Escherichia coli O157:H7. Appl. Environ. Microbiol. 73, 4484-4490. doi: 10.1128/AEM.00340-07

Cantón, R., and Morosini, M. I. (2011). Emergence and spread of antibiotic resistance following exposure to antibiotics. FEMS Microbiol. Rev. 35, 977-991. doi: 10.1111/j.1574-6976.2011.00295.x

Chan, A. C., Ager, D., and Thompson, I. P. (2013). Resolving the mechanism of bacterial inhibition by plant secondary metabolites employing a combination of whole-cell biosensors. J. Microbiol. Methods 93, 209-217. doi: 10.1016/j.mimet.2013.03.021

Charpentier, E., and Tuomanen, E. (2000). Mechanisms of antibiotic resistance and tolerance in Streptococcus pneumoniae. Microbes Infect. 2, 1855-1864. doi: 10.1016/S1286-4579(00)01345-9

Chueca, B., Pagán, R., and García-Gonzalo, D. (2014a). Differential mechanism of Escherichia coli inactivation by $(+)$-limonene as a function of cell physiological state and drug's concentration. PLOS ONE 9:e94072. doi: 10.1371/journal.pone.0094072

Chueca, B., Pagán, R., and García-Gonzalo, D. (2014b). Oxygenated monoterpenes citral and carvacrol cause oxidative damage in Escherichia coli without the involvement of tricarboxylic acid cycle and Fenton reaction. Int. J. Food Microbiol. 189, 126-131. doi: 10.1016/j.ijfoodmicro.2014. 08.008

Chueca, B., Pagán, R., and García-Gonzalo, D. (2015). Transcriptomic analysis of Escherichia coli MG1655 cells exposed to pulsed electric fields. Innov. Food Sci. Emerg. Technol. 29, 78-86. doi: 10.1016/j.ifset.2014.09.003

Clinical and Laboratory Standards Institute (2012). Methods for Dilution Antimicrobial Susceptibility Tests for Bacteria that Grow Aerobically; Approved Standard-9th Edn. CLSI Document M07-A9. Wayne, PA: Clinical and Laboratory Standards Institute.

Collignon, P. J. (2002). 11: antibiotic resistance. Med. J. Aust. 177, 325-329. Available online at: https://www.mja.com.au/journal/2002/177/6/11antibiotic-resistance
$\mathrm{BC}$ with a grant to carry out this investigation. $\mathrm{NN}$ wishes to thank the Coordenação de Aperfeiçoamento de Pessoal de Nível Superior (CAPES-Brazil) for the scholarship granted by the "Science without Borders" Program (Grant 99999.012372/201307). The authors also wish to thank Stanley Hanks (translator) for having revised and proofread the final version of this manuscript.

Condón, S., Palop, A., Raso, J., and Sala, F. J. (1996). Influence of the incubation temperature after heat treatment upon the estimated heat resistance values of spores of Bacillus subtilis. Lett. Appl. Microbiol. 22, 149-152. doi: 10.1111/j.1472-765X.1996.tb01130.x

Corona, F., and Martínez, J. (2013). Phenotypic resistance to antibiotics. Antibiot 2, 237. doi: 10.3390/antibiotics2020237

Dubois-Brissonnet, F., Naïtali, M., Mafu, A. A., and Briandet, R. (2011). Induction of fatty acid composition modifications and tolerance to biocides in Salmonella enterica serovar Typhimurium by plant-derived terpenes. Appl. Environ. Microbiol. 77, 906-910. doi: 10.1128/AEM.01480-10

Espina, L., Condón, S., Pagán, R., and García-Gonzalo, D. (2014). Synergistic effect of orange essential oil or (+)-limonene with heat treatments to inactivate Escherichia coli $\mathrm{O} 157: \mathrm{H} 7$ in orange juice at lower intensities while maintaining hedonic acceptability. Food Bioprocess. Tech. 7, 471-481. doi: 10.1007/s11947013-1076-x

Espina, L., Gelaw, T. K., De Lamo-Castellví, S., Pagán, R., and García-Gonzalo, D. (2013). Mechanism of bacterial inactivation by $(+)$-limonene and its potential use in food preservation combined processes. PLoS ONE 8:e56769. doi: 10.1371/journal.pone.0056769

Fridman, O., Goldberg, A., Ronin, I., Shoresh, N., and Balaban, N. Q. (2014). Optimization of lag time underlies antibiotic tolerance in evolved bacterial populations. Nature 513, 418-421. doi: 10.1038/nature13469

Friedman, M. (2015). Antibiotic-resistant bacteria: prevalence in food and inactivation by food-compatible compounds and plant extracts. J. Agric. Food Chem. 63, 3805-3822. doi: 10.1021/acs.jafc.5b00778

Friedman, M., Henika, P. R., and Mandrell, R. E. (2002). Bactericidal activities of plant essential oils and some of their isolated constituents against Campylobacter jejuni, Escherichia coli, Listeria monocytogenes, and Salmonella enterica. J. Food Prot. 65, 1545-1560. Available online at: http://www. ingentaconnect.com/content/iafp/jfp/2002/00000065/00000010/art00004

García, D., Gómez, N., Raso, J., and Pagán, R. (2005). Bacterial resistance after pulsed electric fields depending on the treatment medium pH. Innov. Food Sci. Emerg. Technol. 6, 388-395. doi: 10.1016/j.ifset.2005.04.003

Gibson, A. M., Bratchell, N., and Roberts, T. A. (1988). Predicting microbialgrowth - growth-responses of salmonellae in a laboratory medium as affected by $\mathrm{pH}$, sodium-chloride and storage-temperature. Int. J. Food Microbiol. 6, 155-178. doi: 10.1016/0168-1605(88)90051-7

Giraud, A., Matic, I., Tenaillon, O., Clara, A., Radman, M., Fons, M., et al. (2001). Costs and benefits of high mutation rates: adaptive evolution of bacteria in the mouse gut. Science 291, 2606-2608. doi: 10.1126/science.10 56421

Gomes Neto, N. J., Luz Ida, S., Tavares, A. G., Honório, V. G., Magnani, M., and De Souza, E. L. (2012). Rosmarinus officinalis L. essential oil and its majority compound 1,8-cineole at sublethal amounts induce no direct and cross protection in Staphylococcus aureus ATCC 6538. Foodborne Pathog. Dis. 9, 1071-1076. doi: 10.1089/fpd.2012.1258

Gomes Neto, N. J., Magnani, M., Chueca, B., García-Gonzalo, D., Pagán, R., and De Souza, E. L. (2015). Influence of general stress-response alternative sigma factors $\sigma^{S}$ (RpoS) and $\sigma^{B}(\operatorname{SigB})$ on bacterial tolerance to the essential oils from Origanum vulgare L. and Rosmarinus officinalis L. and pulsed electric fields. Int. J. Food Microbiol. 211, 32-37. doi: 10.1016/j.ijfoodmicro.2015. 06.030

Gutierrez, A., Laureti, L., Crussard, S., Abida, H., Rodríguez-Rojas, A., Blázquez, J., et al. (2013). $\beta$-lactam antibiotics promote bacterial mutagenesis via an RpoS-mediated reduction in replication fidelity. Nat. Commun. 4, 1610. doi: $10.1038 /$ ncomms 2607 
Hammer, K. A., Carson, C. F., and Riley, T. V. (2012). Effects of Melaleuca alternifolia (tea tree) essential oil and the major monoterpene component terpinen-4-ol on the development of single- and multistep antibiotic resistance and antimicrobial susceptibility. Antimicrob. Agents Chemother. 56, 909-915. doi: 10.1128/AAC.05741-11

Hauben, K. J. A., Bartlett, D. H., Soontjens, C. C. F., Cornelis, K., Wuytack, E. Y., and Michiels, C. W. (1997). Escherichia coli mutants resistant to inactivation by high hydrostatic pressure. Appl. Environ. Microbiol. 63, 945-950.

Hengge-Aronis, R. (2011). "The general stress response in Gram-negative bacteria," in Bacterial Stress Responses, 2nd Edn., eds G. Storz and R. Hengge (Washington, DC: ASM Press), 251-289. doi: 10.1128/9781555816841.ch15

Hyldgaard, M., Mygind, T., and Meyer, R. L. (2012). Essential oils in food preservation: mode of action, synergies, and interactions with food matrix components. Front. Microbiol. 3:12. doi: 10.3389/fmicb.2012.00012

Jones, T. H., Vail, K. M., and McMullen, L. M. (2013). Filament formation by foodborne bacteria under sublethal stress. Int. J. Food Microbiol. 165, 97-110. doi: 10.1016/j.ijfoodmicro.2013.05.001

Kieboom, J., Kusumaningrum, H. D., Tempelaars, M. H., Hazeleger, W. C., Abee, T., and Beumer, R. R. (2006). Survival, elongation, and elevated tolerance of Salmonella enterica serovar Enteritidis at reduced water activity. J. Food Prot. 69, 2681-2686. Available online at: http://www.ingentaconnect.com/content/ $\mathrm{iafp} / \mathrm{jfp} / 2006 / 00000069 / 00000011 /$ art00015

Kohanski, M. A., Depristo, M. A., and Collins, J. J. (2010). Sublethal antibiotic treatment leads to multidrug resistance via radical-induced mutagenesis. Mol. Cell 37, 311-320. doi: 10.1016/j.molcel.2010.01.003

Kohanski, M. A., Dwyer, D. J., Hayete, B., Lawrence, C. A., and Collins, J. J. (2007). A common mechanism of cellular death induced by bactericidal antibiotics. Cell 130, 797-810. doi: 10.1016/j.cell.2007.06.049

Lenski, R. E., Rose, M. R., Simpson, S. C., and Tadler, S. C. (1991). Long-Term experimental evolution in Escherichia coli. I. Adaptation and divergence during 2,000 generations. Am. Nat. 138, 1315-1341. doi: 10.1086/2 85289

Llana-Ruiz-Cabello, M., Gutiérrez-Praena, D., Puerto, M., Pichardo, S., Jos, Á., and Cameán, A. M. (2015). In vitro pro-oxidant/antioxidant role of carvacrol, thymol and their mixture in the intestinal Caco-2 cell line. Toxicol. In Vitro 29, 647-656. doi: 10.1016/j.tiv.2015.02.006

Luz, I. D. S., Gomes Neto, N. J., Tavares, A. G., Nunes, P. C., Magnani, M., and De Souza, E. L. (2012a). Evidence for lack of acquisition of tolerance in Salmonella enterica serovar Typhimurium ATCC 14028 after exposure to subinhibitory amounts of Origanum vulgare L. essential oil and carvacrol. Appl. Environ. Microbiol. 78, 5021-5024. doi: 10.1128/AEM.00605-12

Luz, I. D. S., Neto, N. J. G., Tavares, A. G., Magnani, M., and De Souza, E. L. (2012b). Exposure of Listeria monocytogenes to sublethal amounts of Origanum vulgare $\mathrm{L}$. essential oil or carvacrol in a food-based medium does not induce direct or cross protection. Food Res. Int. 48, 667-672. doi: 10.1016/j.foodres.2012.05.026

Mackey, B. M., Miles, C. A., Parsons, S. E., and Seymour, D. A. (1991). Thermal denaturation of whole cells and cell components of Escherichia coli examined by differential scanning calorimetry. J. Gen. Microbiol. 137, 2361-2374. doi: 10.1099/00221287-137-10-2361

McMahon, M. A., Tunney, M. M., Moore, J. E., Blair, I. S., Gilpin, D. F., and McDowell, D. A. (2008). Changes in antibiotic susceptibility in staphylococci habituated to sub-lethal concentrations of tea tree oil (Melaleuca alternifolia). Lett. Appl. Microbiol. 47, 263-268. doi: 10.1111/j.1472-765X.2008. 02420.x

McMahon, M. A., Xu, J., Moore, J. E., Blair, I. S., and McDowell, D. A. (2007). Environmental stress and antibiotic resistance in food-related pathogens. Appl. Environ. Microbiol. 73, 211-217. doi: 10.1128/AEM.00578-06
Patrignani, F., Iucci, L., Belletti, N., Gardini, F., Guerzoni, M. E., and Lanciotti, R. (2008). Effects of sub-lethal concentrations of hexanal and 2-(E)-hexenal on membrane fatty acid composition and volatile compounds of Listeria monocytogenes, Staphylococcus aureus, Salmonella enteritidis and Escherichia coli. Int. J. Food Microbiol. 123, 1-8. doi: 10.1016/j.ijfoodmicro.2007.09.009

Raso, J., Álvarez, I., Condón, S., and Sala, F. J. (2000). Predicting inactivation of Salmonella senftenberg by pulsed electric fields. Innov. Food Sci. Emerg. Technol. 1, 21-30. doi: 10.1016/S1466-8564(99)00005-3

Renzoni, A., Andrey, D. O., Jousselin, A., Barras, C., Monod, A., Vaudaux, P., et al. (2011). Whole genome sequencing and complete genetic analysis reveals novel pathways to glycopeptide resistance in Staphylococcus aureus. PLoS ONE 6:e21577. doi: 10.1371/journal.pone.0021577

Rosche, W. A., and Foster, P. L. (2000). Determining mutation rates in bacterial populations. Methods 20, 4-17. doi: 10.1006/meth.1999.0901

Rota, C., Carramiñana, J. J., Burillo, J., and Herrera, A. (2004). In vitro antimicrobial activity of essential oils from aromatic plants against selected foodborne pathogens. J. Food Prot. 67, 1252-1256. Available online at: http://www.ingentaconnect.com/content/iafp/jfp/2004/00000067/00000006/ $\operatorname{art} 00028$

Sagarzazu, N., Cebrián, G., Pagán, R., Condón, S., and Mañas, P. (2013). Emergence of pulsed electric fields resistance in Salmonella enterica serovar Typhimurium SL1344. Int. J. Food Microbiol. 166, 219-225. doi: 10.1016/j.ijfoodmicro.2013.07.001

Somolinos, M., García, D., Condón, S., Mackey, B., and Pagán, R. (2010). Inactivation of Escherichia coli by citral. J. Appl. Microbiol. 108, 1928-1939. doi: 10.1111/j.1365-2672.2009.04597.x

Thi, T. D., López, E., Rodríguez-Rojas, A., Rodríguez-Beltrán, J., Couce, A., Guelfo, J. R., et al. (2011). Effect of recA inactivation on mutagenesis of Escherichia coli exposed to sublethal concentrations of antimicrobials. J. Antimicrob. Chemother. 66, 531-538. doi: 10.1093/jac/dkq496

Tsuchido, T., Katsui, N., Takeuchi, A., Takano, M., and Shibasaki, I. (1985). Destruction of the outer membrane permeability barrier of Escherichia coli by heat treatment. Appl. Environ. Microbiol. 50, 298-303.

Ultee, A., Bennik, M. H. J., and Moezelaar, R. (2002). The phenolic hydroxyl group of carvacrol is essential for action against the food-borne pathogen Bacillus cereus. Appl. Environ. Microbiol. 68, 1561-1568. doi: 10.1128/AEM.68.4.15611568.2002

Vidovic, S., Mangalappalli-Illathu, A. K., and Korber, D. R. (2011). Prolonged cold stress response of Escherichia coli $\mathrm{O} 157$ and the role of rpoS. Int. J. Food Microbiol. 146, 163-169. doi: 10.1016/j.ijfoodmicro.2011.02.018

Vidovic, S., Mangalappalli-Illathu, A. K., Xiong, H., and Korber, D. R. (2012). Heat acclimation and the role of RpoS in prolonged heat shock of Escherichia coli O157. Food Microbiol. 30, 457-464. doi: 10.1016/j.fm.2011.12.029

Visvalingam, J., Gill, C. O., and Holley, R. A. (2012). The viabilities of cells in cultures of Escherichia coli growing with formation of filaments at $6^{\circ}$ C. Int. J. Food Microbiol. 153, 129-134. doi: 10.1016/j.ijfoodmicro.2011. 10.027

Conflict of Interest Statement: The authors declare that the research was conducted in the absence of any commercial or financial relationships that could be construed as a potential conflict of interest.

Copyright (๑ 2016 Chueca, Berdejo, Gomes-Neto, Pagán and García-Gonzalo. This is an open-access article distributed under the terms of the Creative Commons Attribution License (CC BY). The use, distribution or reproduction in other forums is permitted, provided the original author(s) or licensor are credited and that the original publication in this journal is cited, in accordance with accepted academic practice. No use, distribution or reproduction is permitted which does not comply with these terms. 